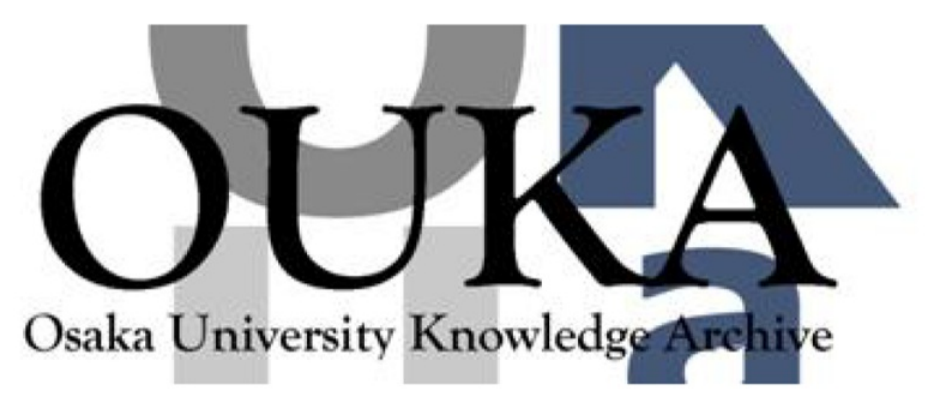

\begin{tabular}{|c|c|}
\hline Title & $\begin{array}{l}\text { Short-distance behavior of the Bethe-Salpeter } \\
\text { amplitude }\end{array}$ \\
\hline Author (s) & Higashijima, Kiyoshi \\
\hline Citation & $\begin{array}{l}\text { Physical review. Third series. D, Particles and } \\
\text { fields. 18(6) p. } 2128-p .2133\end{array}$ \\
\hline Issue Date & $1978-09$ \\
\hline oaire:version & VoR \\
\hline URL & ht tps://hdl. handle. net/11094/3470 \\
\hline rights & $\begin{array}{l}\text { Higashijima, Kiyoshi, Physical review. Third } \\
\text { series. D, Particles and fields, 18,6, } 2128- \\
2133,1978-09 \text {. "Copyright } 1978 \text { by the American } \\
\text { Physical Society." }\end{array}$ \\
\hline Note & \\
\hline
\end{tabular}

Osaka University Knowledge Archive : OUKA

https://ir. Library. osaka-u. ac. jp/

0saka University 


\title{
Short-distance behavior of the Bethe-Salpeter amplitude
}

\author{
Kiyoshi Higashijima* \\ Department of Physics, Purdue University, West Lafayette, Indiana 47907
}

(Received 8 May 1978)

\begin{abstract}
A simple relation between the Bethe-Salpeter amplitude at the origin and a certain renormalization constant is established. A homogeneous Bethe-Salpeter equation is shown to have a continuous spectrum when the amplitude diverges at the origin. A prescription to extract the bound states from the continuous spectrum is proposed for the asymptotically free theory.
\end{abstract}

\section{INTRODUCTION}

The short-distance behavior of the Bethe-Salpeter (BS) amplitude is interesting for several reasons: In nonrelativistic quantum mechanics, the wave function at the origin is intimately connected with observable quantities such as the pion decay constant or the vector-meson coupling to the photon. The behavior of the BS amplitude at the origin plays a crucial role in the study of the asymptotic behavior of the form factors or scattering amplitudes; for example, one of the basic assumptions of the Brodsky-Farrar quark counting rule is the finiteness of the $\mathrm{BS}$ amplitude at the origin. ${ }^{1}$

Many authors ${ }^{2}$ have discussed the short-distance behavior of the BS amplitude, either by solving the BS equation in the ladder approximation or by making an operator-product expansion and a renormalization-group argument. Explicit calculations based on the se approaches sometimes lead to the divergence of the BS amplitudes at the origin. On the other hand, Nishijima, Sato, and Ezawa $^{3}$ (NSE) have proved the finiteness of the BS amplitude at the origin on quite general grounds. In some cases, therefore, there are contradictions between the results of explicit calculations and the NSE theorem. Although the NSE theorem is surely true when the theory is superrenormalizable, it seems to require modification by taking renormalization effects into consideration when the theory is renormalizable.

In this paper, we obtain a general theorem on the BS amplitude at the origin. The theorem reconciles the contradiction between the explicit calculations and the NSE theorem. Our essential assumption is the renormalizability of the theory, so that our new theorem is not valid if the theory is nonrenormalizable. We also discuss the continuous spectrum of the homogeneous BS equation which appears when the BS amplitude diverges at the origin.

In the next section, the renormalization of composite operators is briefly reviewed. In Sec. III the general theorem on the BS amplitude is derived. Section $I V$ is devoted to the discussion of the continuous spectrum of the homogeneous BS equation. In Sec. $\mathrm{V}$ a generalization of the NSE theorem is discussed, after a short review of its derivation.

\section{RENORMALIZATION OF COMPOSITE OPERATORS}

In this section, we shall recapitulate the renormalization of composite operators,

$$
\Theta(x) \equiv \bar{\Psi}(x) \theta \Psi(x)
$$

where $\theta$ denotes some numerical matrix acting on spinor indices or internal-symmetry indices. The canonical dimension of $\Theta(x)$ is 3 . We assume that our theory is renormalizable, and that there is no other operator having the same quantum number as $\Theta(x)$ with dimensionality less than or equal to 3. On these assumptions, the composite operator $\Theta(x)$ is $\mathrm{known}^{4}$ to be multiplicatively renormalizable; in other words, any matrix element of the renormalized operator defined by

$$
\Theta_{R}(x)=Z_{\theta}{ }^{-1} \Theta(x)
$$

can be made finite by a suitable choice of the renormalization constant $Z_{\theta}$. In perturbation theory, $Z_{\theta}{ }^{-1}$ has logarithmic divergences as the cutoff $\Lambda$ tends to infinity.

The proper vertex $\Gamma^{\theta}$ is defined by

$$
\begin{aligned}
S(p) & \Gamma^{\theta}\left(p, p^{\prime}\right) S\left(p^{\prime}\right) \\
& \equiv \int d^{4} x \int d^{4} y e^{i\left(p \cdot x-p^{\prime} \cdot y\right)}\langle 0|T[\Psi(x) \bar{\Psi}(y) \Theta(0)]| 0\rangle,
\end{aligned}
$$

where $S(p)$ denotes the fermion propagator. The renormalization constant $Z_{1 \theta}$ for the proper vertex is introduced by

$$
\Gamma^{\theta}\left(p, p^{\prime}\right)=Z_{1 \theta}{ }^{-1} \Gamma_{R}^{\theta}\left(p, p^{\prime}\right),
$$

where the renormalized vertex $\Gamma_{R}^{\theta}$ is defined by a similar equation to Eq. (2.3) for renormalized quantities. Substitution of $S(p)=Z_{2} S_{R}(p)$, 
$\Psi(x)=Z_{2}^{1 / 2} \Psi_{R}(x)$, Eqs. (2.2) and (2.4) into Eq. (2.3) leads to a relation between $Z_{\theta}$ and $Z_{1 \theta}$,

$$
Z_{\theta}=Z_{1 \theta}{ }^{-1} Z_{2} \text {. }
$$

If $\Theta(x)$ is conserved, there is no renormalization for $\Theta(x)$, by virtue of the Ward-Takahashi identity $Z_{1 \theta}=Z_{2}$.

If there are other operators which have the same quantum numbers as $\Theta(x)$ and dimensionality less than or equal to 3 , we would need additive renormalizations. As an example, let us introduce an elementary boson $\varphi(x)$ carrying the same quantum number as $\Theta(x)$ in our theory. For simplicity, we assume $\Theta(x)$ is scalar or pseudoscalar. Since $\varphi(x)$ has dimension 1, Eq. (2.2) is no longer valid. Instead we have

$$
\Theta(x)=Z_{\theta} \Theta_{R}(x)+a \varphi_{R}(x)+b\left(\square+M_{R}^{2}\right) \varphi_{R}(x) .
$$

In perturbation theory, $a$ diverges quadratically and $b$ logarithmically.

\section{SHORT-DISTANCE BEHAVIOR OF THE BS AMPLITUDE}

In order to derive the relation between a renormalization constant and the short-distance behavior of the BS amplitude, we will imagine all the renormalization constants are finite. Let us first recall the definition of the unrenormalized composite operator (2.1):

$$
\lim _{\xi \rightarrow 0} T \bar{\Psi}\left(x-\frac{1}{2} \xi\right) \theta \Psi\left(x+\frac{1}{2} \xi\right)=\Theta(x),
$$

where the limit $\xi \rightarrow 0$ is understood to be taken from a spacelike direction. It should be noted that a similar relation as Eq. (3.1) does not hold for the renormalized quantities. By substituting Eq. (2.2) and $\Psi(x)=Z_{2}{ }^{1 / 2} \Psi_{R}(x)$ into Eq. (3.1), we get a corresponding relation for the renormalized quantities,

$$
\lim _{\xi \rightarrow 0} T \bar{\Psi}_{R}\left(x-\frac{1}{2} \xi\right) \theta \Psi_{R}\left(x+\frac{1}{2} \xi\right)=Z_{1 \theta}{ }^{-1} \Theta_{R}(x),
$$

where use has been made of Eq. (2.5). After use of the definition of the renormalized BS amplitude for a bound state $|P\rangle$,

$$
\begin{aligned}
\chi_{R}(x ; P) & \equiv\left\langle 0\left|T \Psi_{R}\left(\frac{1}{2} x\right) \bar{\Psi}_{R}\left(-\frac{1}{2} x\right)\right| P\right\rangle \\
& =\int \frac{d^{4} q}{(2 \pi)^{4}} \chi_{R}(q ; P) e^{-i q \cdot x},
\end{aligned}
$$

Eq. (3.2) gives

$$
\lim _{x \rightarrow 0} \operatorname{Tr}\left[\theta \chi_{R}(x ; P)\right]=-Z_{1 \theta^{-1}} c_{R},
$$

where

$$
c_{R} \equiv\left\langle 0\left|\Theta_{R}(0)\right| P\right\rangle
$$

is a finite constant by definition. Note that the equal-time commutator which appears when we interchange $\Psi_{R}$ and $\bar{\Psi}_{R}$ does not contribute to the matrix element. We also note that in momentum space Eq. (3.4) reads

$$
\int \frac{d^{4} q}{(2 \pi)^{4}} \operatorname{Tr}\left[\theta \chi_{R}(q ; P)\right]=-Z_{1 \theta}{ }^{-1} c_{R} .
$$

On the basis of Eq. (3.4), we can conclude that the BS amplitude $\operatorname{Tr}\left[\theta \chi_{R}(x ; P)\right]$ is finite at the origin, as long as the renormalization constant $Z_{1 \theta}{ }^{-1}$ of the vertex $\Gamma^{\theta}$ is finite.

When $Z_{1 \theta}$ vanishes, the above derivation of Eq. (3.4) is no longer valid, since Eq. (3.2) is meaningless. Nevertheless, we can give a heuristic interpretation to Eq. (3.4) by introducing a cutoff $\Lambda$. In the cutoff theory, the BS amplitude at $x$ smaller than $\Lambda^{-1}$ is meaningless. Therefore, the limit $x \rightarrow 0$ in Eq. (3.4) should be interpreted as $x \rightarrow \Lambda^{-1}$. Correspondingly, $Z_{1 \theta^{-1}}$ in Eq. (3.4) should be regarded as the renormalization constant $Z_{1 \theta}{ }^{-1}(\Lambda)$ in the cutoff theory, that is, Eq. (3.4) can be interpreted as follows:

$$
\lim _{x \rightarrow \Lambda^{-1}} \operatorname{Tr}\left[\theta \chi_{R}(x ; P)\right]=-Z_{1 \theta}^{-1}(\Lambda) c_{R}
$$

when $Z_{1 \theta} \equiv \lim _{\Lambda \rightarrow \infty} Z_{1 \theta}(\Lambda)=0$. In the cutoff theory, the renormalization constant $Z_{1 \theta}(\Lambda)$ satisfies a homogeneous Callan-Symanzik ${ }^{5}$ equation,

$$
\begin{aligned}
{\left[m_{R} \frac{\partial}{\partial m_{R}}+\beta\left(g_{R}\right) \frac{\partial}{\partial g_{R}}+\right.} & \left.\gamma_{\theta}\left(g_{R}\right)-2 \gamma_{\Psi}\left(g_{R}\right)\right] \\
& \times Z_{1 \theta}\left(g_{R}, \frac{\Lambda}{m_{R}}\right)=0
\end{aligned}
$$

when $Z_{1 \theta}$ is expressed in terms of $g_{R}, m_{R}$, and $\Lambda$. If our theory satisfies either asymptotic freedom or broken scale invariance, Eq. (3.7) can be solved asymptotically and gives the following results:

(a) If $\beta\left(g_{R}\right)=-a g_{R}{ }^{3}+\cdots$ with $a>0$, then

$$
Z_{1 \theta}{ }^{-1}(\Lambda) \propto\left(\ln \Lambda^{2}\right)^{-\left(2 b_{\Psi}-b_{\theta}\right) / a},
$$

where $\gamma_{\Psi}\left(g_{R}\right)=b_{\Psi} g_{R}^{2}+\cdots$ and $\gamma_{\theta}\left(g_{R}\right)=b_{\theta} g_{R}{ }^{2}+\cdots$. This relation together with Eq. (3.6) gives

$$
\operatorname{Tr}\left[\theta \chi_{R}(x ; P)\right] \underset{x \rightarrow 0}{\sim}\left(\ln \frac{1}{x^{2}}\right)^{-\left(2 b_{\Psi}-b_{\theta}\right) / a}
$$

(b) If $\beta\left(g_{R}\right)$ has a fixed point such that $\beta\left(g_{\infty}\right)=0$ and $\beta^{\prime}\left(g_{\infty}\right)<0$, then

$$
Z_{1 \theta}{ }^{-1}(\Lambda) \propto\left(\Lambda^{2}\right)^{\left[2 \gamma_{\Psi}\left(g_{\infty}\right)-\gamma_{\theta}\left(g_{\infty}\right)\right] / 2},
$$

this implies

$$
\operatorname{Tr}\left[\theta \chi_{R}(x ; P)\right] \underset{x \rightarrow 0}{\sim}\left(1 / x^{2}\right)^{\left[2 \gamma_{\Psi}\left(g_{\infty}\right)-\gamma_{\theta}\left(g_{\infty}\right)\right] / 2} .
$$

Even when $Z_{1 \theta}$ vanishes, a correct treatment of Eq. (3.2) is given by a Wils on ${ }^{6}$ operator-product expansion,

$T \bar{\Psi}_{R}\left(x-\frac{1}{2} \xi\right) \theta \Psi_{R}\left(x+\frac{1}{2} \xi\right)=Z(\xi) \Theta_{R}(x)+\cdots$, 
where an irrelevant vacuum expectation value has been subtracted. From Eqs. (3.3) and (3.13), we find

$$
\operatorname{Tr}\left[\theta \chi_{R}(x ; P)\right] \underset{x \rightarrow 0}{\sim}-Z(x) c_{R}+\cdots .
$$

By comparing Eqs. (3.7) and (3.14), we obtain a heuristic interpretation of a Wilson coefficient function $Z(x)$,

$$
\lim _{x \rightarrow \Lambda^{-1}} Z(x)=Z_{1 \theta^{-1}}(\Lambda) \text {. }
$$

Equation (3.14) together with the renormalizationgroup argument applied to $Z(\xi)$ reproduces our previous results (3.10) and (3.12) in those two special cases.

Some comments should be added:

(1) If there is a gauge field $A_{\mu}(x)$ in our theory, special care should be exercised to define composite operators. ${ }^{7}$ In order to ensure the gauge invariance, we must insert $\exp \left(i \int_{x+\xi / 2}^{x-\xi / 2} A_{\mu} d x^{\mu}\right)$ between $\bar{\Psi}\left(x-\frac{1}{2} \xi\right)$ and $\Psi\left(x+\frac{1}{2} \xi\right)$ in Eq. (3.1). We are, however, assuming that there is no other operator which has the same quantum numbers as $\Theta(x)$ and dimensionality less than or equal to 3 , so that the contribution of the line integral vanishes in the limit of $\xi \rightarrow 0$ for these $\Theta(x)$. For example, consider the quark model with colored gauge fields. The assumption forces us to choose such $\boldsymbol{\Theta}(x)$ as a flavor nonsinglet. Since we cannot make local flavor nonsinglet operators out of $A_{\mu}(x)$, the line integral does not contribute in Eq. (3.1) for these $\Theta(x)$. Our main result, Eqs. (3.4) or (3.7), cannot be applied to the flavor-singlet $\theta$.

(2) In nonrelativistic quantum mechanics, the wave function at the orig in is considered to be an observable quantity; it represents the pion decay constant or the coupling of a vector meson with a photon. On the other hand, the BS amplitude at the origin does not contain this information, because of renormalization effects. Therefore, the divergence of the BS amplitude does not imply any divergence of physical quantities. The pion decay constant or the vector-meson coupling with a photon is expressed in terms of $c_{R} \equiv\left\langle 0\left|\Theta_{R}(0)\right| P\right\rangle$, which is finite.

(3) If there is an elementary boson field $\varphi_{R}(x)$ in our theory, Eq. (3.4) or (3.7) is no longer valid. In this case, Eq. (2.6) yields

$$
\begin{aligned}
\lim _{x \rightarrow \Lambda^{-1}} \operatorname{Tr}\left[\theta \chi_{R}(x ; P)\right] & =-Z_{1 \theta}^{-1}(\Lambda) c_{R} \\
& -Z_{2}^{-1}(\Lambda)\left[a(\Lambda)+\left(M_{R}^{2}-M_{B}{ }^{2}\right) b(\Lambda)\right] \\
& \times\left\langle 0\left|\varphi_{R}(0)\right| P\right\rangle,
\end{aligned}
$$

where $M_{R}$ denotes the mass of $\varphi_{R}$ and $M_{B}$ the mass of the bound state $|P\rangle$. Since $a(\Lambda)$ diverges quadratically in perturbation theory, the $\mathrm{BS}$ amplitude seems to be more divergent in this case than before.

(4) Several years ago, Brodsky and Farrar ${ }^{1}$ proposed their power counting law for the scattering amplitudes. One of their basic assumptions is the finiteness of the BS amplitude at the origin, namely,

$$
\int \frac{d^{4} q}{(2 \pi)^{4}} \chi_{R}(q ; P)=\text { finite } .
$$

As a result of Eq. (3.6), this condition is satisfied unless $Z_{1 \theta}$ vanishes.

(5) Equation (3.7) has an analogy in the KällénLehmann sum rule for the renormalized propagator

$$
\lim _{p \rightarrow \infty} \operatorname{Tr}\left[\not p S_{R}(p)\right]=4 i Z_{2}{ }^{-1},
$$

which is interpreted as

$$
\lim _{p \rightarrow \Lambda} \operatorname{Tr}\left[\not p S_{R}(p)\right]=4 i Z_{2}^{-1}(\Lambda)
$$

when $Z_{2}=\lim _{\Lambda \rightarrow \infty} Z_{2}(\Lambda)$ vanishes.

\section{CONTINUOUS SPECTRUM OF THE HOMOGENEOUS BS EQUATION}

The vanishing of $Z_{1 \theta}$ causes a certain difficulty ${ }^{8}$ in understanding the solution of a homogeneous BS equation. The homogeneous BS equation for a bound state with mass $M_{B}$ reads

$$
\begin{aligned}
S_{R}^{-1}\left(q+\frac{1}{2} P\right) \chi_{R}(q ; P) S_{R}^{-1}\left(q-\frac{1}{2} P\right) & \\
& =\int \frac{d^{4} q^{\prime}}{(2 \pi)^{4}} I_{R}\left(q, q^{\prime} ; P\right) \chi_{R}\left(q^{\prime} ; P\right),
\end{aligned}
$$

where $I_{R}\left(q, q^{\prime} ; P\right)$ denotes the renormalized twoparticle irreducible BS kernel. Equation (4.1) is expected to have solutions for discrete values of $P^{2}$, which gives the mass $M_{B}{ }^{2}$ of bound states. When $Z_{1 \theta}$ vanishes, however, this is not the case; the homogeneous BS equation has solutions for any $P^{2}$. In order to see this, let us study the nonamputated vertex function

$$
\Phi^{\theta}(q ; P) \equiv S(p) \Gamma^{\theta}\left(p, p^{\prime}\right) S\left(p^{\prime}\right),
$$

where

$$
p=q+\frac{1}{2} P, \quad p^{\prime}=q-\frac{1}{2} P .
$$

The inhomogeneous BS equation satisfied by the unrenormalized vertex $\Phi^{\theta}(q ; P)$ reads

$$
\begin{aligned}
S^{-1}\left(q+\frac{1}{2} P\right) & \Phi^{\theta}(q ; P) S^{-1}\left(q-\frac{1}{2} P\right) \\
& =\theta+\int \frac{d^{4} q^{\prime}}{(2 \pi)^{4}} I\left(q, q^{\prime} ; P\right) \Phi^{\theta}\left(q^{\prime} ; P\right) .
\end{aligned}
$$

After renormalization,

$$
\Phi^{\theta}(q ; P)=Z_{2} Z_{\theta} \Phi_{R}{ }^{\theta}(q ; P)
$$


and

$$
I\left(q, q^{\prime} ; P\right)=Z_{2}^{-2} I_{R}\left(q, q^{\prime} ; P\right) .
$$

Equation (4.3) can be rewritten as

$$
\begin{aligned}
S_{R}^{-1}(q+ & \left.\frac{1}{2} P\right) \Phi_{R}^{\theta}(q ; P) S_{R}^{-1}\left(q-\frac{1}{2} P\right) \\
& =Z_{1 \theta} \theta+\int \frac{d^{4} q^{\prime}}{(2 \pi)^{4}} I_{R}\left(q, q^{\prime} ; P\right) \Phi_{R}^{\theta}\left(q^{\prime} ; P\right) .
\end{aligned}
$$

Since the vertex function should exist for any $P^{2}$, Eq. (4.4) must have solutions for any $P^{2}$ except for $P^{2}=M_{B}{ }^{2}$. When $Z_{1 \theta}$ vanishes, Eq. (4.4) reduces to the homogeneous BS equation. Therefore, it can be concluded that the homogeneous BS equation must have a continuous spectrum corresponding to the renormalized vertex when $Z_{1 \theta}$ vanishes. If we combine this with the result of the preceding section, it turns out that the homogeneous $\mathrm{BS}$ equation possesses a continuous spectrum when the $\mathrm{BS}$ amplitude diverges at the origin.

When $Z_{1 \theta}$ vanishes, some ad hoc boundary condition is required to extract the discrete spectrum from the continuous spectrum; otherwise, we cannot get the energy levels of bound states by solving the $\mathrm{BS}$ equation. It is $\mathrm{known}^{8,9}$ that the required boundary condition is given by the normalization condition in the ladder approximation. The most general form of the normalization condition ${ }^{10}$ can be written as

$$
\begin{aligned}
-i \int \frac{d^{4} q}{(2 \pi)^{4}} \operatorname{Tr} & {\left[\bar{\chi}_{R}(q ; P) S_{R}^{-1}\left(q+\frac{1}{2} P\right) \chi_{R}(q ; P)\right.} \\
& \left.\times S_{R}^{-1}\left(q-\frac{1}{2} P\right)\right]=\left.\frac{d}{d \lambda} M_{B}^{2}(\lambda)\right|_{\lambda=1},
\end{aligned}
$$

where $\lambda$ is an artificially introduced parameter multiplying $I_{R}$ in Eq. (4.1). We will discuss below the restriction demanded by the finiteness of the normalization integral (4.5).

In order to see whether or not the finiteness of Eq. (4.5) rejects the continuous spectrum representing the renormalized vertex, we have to know the short-distance behavior of the vertex function. Let us introduce the Fourier transform of $\Phi^{\theta}(q ; P)$ with respect to $q$ :

$$
\begin{aligned}
\Phi^{\theta}(\xi ; P) \equiv & \int \frac{d^{4} q}{(2 \pi)^{4}} e^{-i q \xi} \Phi^{\theta}(q ; P) \\
= & \int d^{4} x e^{i P_{x}} \\
& \quad \times\left\langle 0\left|T \Psi\left(x+\frac{1}{2} \xi\right) \bar{\Psi}\left(x-\frac{1}{2} \xi\right) \Theta(0)\right| 0\right\rangle .
\end{aligned}
$$

On applying Eq. (3.1) to Eq. (4.6), we have

$$
\begin{aligned}
\lim _{\xi \rightarrow 0} \operatorname{Tr}\left[\theta \Phi^{\theta}(\xi ; P)\right] & =-\int d^{4} x e^{i P x}\langle 0|T \Theta(x) \Theta(0)| 0\rangle \\
& \equiv-\Pi^{\theta}(P) .
\end{aligned}
$$

Since $\Pi^{\theta}(P)$ has a quadratic divergence, additive renormalizations are also required to renormalize it,

$$
\Pi^{\theta}(P)=Z_{\theta}^{2}\left[A+B P^{2}+\Pi_{R}^{\theta}(P)\right],
$$

where $\Pi_{R}^{\theta}(P)$ represents the finite part. In perturbation theory, $A$ diverges quadratically and $B$ logarithmically. From Eqs. (4.7), (4.8), and $\Phi^{\theta}$ $=Z_{1 \theta}{ }^{-1} Z_{2}{ }^{2} \Phi_{R}^{\theta}$, we find

$$
\begin{aligned}
\lim _{\xi \rightarrow \Lambda^{-1}} \operatorname{Tr}\left[\theta \Phi_{R}^{\theta}(\xi ; P)\right]=-Z_{1 \theta}{ }^{-1}(\Lambda)[ & A(\Lambda)+P^{2} B(\Lambda) \\
& \left.+\Pi_{R}^{\theta}(P)\right] .
\end{aligned}
$$

In a free-field theory, $A(\Lambda) \propto \Lambda^{2}, Z_{1 \theta}$, and $B(\Lambda)$ are constants, so that $\Phi_{R}^{\theta}(x, P)$ behaves like $1 / x^{2}$ at short distances. If we confine our discussion to an asymptotically free theory, this $1 / x^{2}$ behavior is modified, at most by some powers of $\ln \left(1 / x^{2}\right)$ in the exact theory. Therefore, in momentum space, $\Phi_{R}^{\theta}(q ; P)$ behaves as $q^{-2}$, up to a logarithmic factor, when $q \rightarrow \infty$. Since $S_{R}^{-1}(q) \sim \not$, up to a logarithmic factor, when $q \rightarrow \infty$, the normalization integral Eq. (4.5) for the vertex function $\Phi_{R}^{\theta}(q ; P)$ diverges quadratically in the untraviolet region. On the other hand, it converges for the BS amplitude which behaves as $\chi_{R}(q ; P) \sim q^{-4}$, up to a logarithmic factor, as is seen from Eq. (3.10). Consequently, in an asymptotically free theory, we can reject the continuous spectrum of the homogeneous $B S$ equation by requiring the finiteness of the normalization integral. In more general theories, the validity of the above result is not clear.

\section{EXTENSION OF THE NSE THEOREM}

In general, any field operator $\varphi_{c}(x)$ can be chosen as an interpolating field for a bound state $|P\rangle$, as long as it has a nonvanishing matrix element $\left\langle 0\left|\varphi_{c}(x)\right| P\right\rangle$. Although each choice of $\varphi_{c}(x)$ gives a different off-shell extrapolation, all of them lead to the identical $S$ matrix. As a special example, Haag, Nishijima, and Zimmermann ${ }^{11}$ (HNZ) have proposed to construct an interpolating field $\varphi_{c}(x)$ by

$$
\begin{aligned}
\varphi_{c}(x) & =\lim _{\xi \rightarrow 0} \frac{T \bar{\Psi}\left(x-\frac{1}{2} \xi\right) \theta \Psi\left(x+\frac{1}{2} \xi\right)}{\left\langle 0\left|T \bar{\Psi}\left(-\frac{1}{2} \xi\right) \theta \Psi\left(\frac{1}{2} \xi\right)\right| P\right\rangle} \\
& =\frac{\Theta(x)}{\langle 0|\Theta(0)| P\rangle},
\end{aligned}
$$

where use has been made of the definition (3.1). Using Eqs. (2.2) and (3.5), we can express $\varphi_{c}(x)$ in terms of the renormalized quantities, 


$$
\varphi_{c}(x)=c_{R}^{-1} \Theta_{R}(x) .
$$

Thus, in the HNZ construction, the interpolating field $\varphi_{c}(x)$ coincides with the renormalized com- posite operator $\Theta_{R}(x)$ except for the finite multiplicative constant $c_{R}^{-1}$.

Now, we are ready to discuss the $\mathrm{NSE}^{3}$ theorem. They have studied a Green's function defined by

$$
(2 \pi)^{4} \delta^{4}\left(p-p^{\prime}-P\right) S(q ; P) \equiv \int d^{4} x \int d^{4} y \int d^{4} z e^{i\left(p x-p^{\prime} y-P z\right)}\left(M_{B}^{2}+\square_{z}\right)\left\langle 0\left|T\left[\Psi_{R}(x) \Psi_{R}(y) \varphi_{c}(z)\right]\right| 0\right\rangle,
$$

where $2 q=p+p^{\prime}$. Substituting Eq. (5.2) into Eq. (5.3) and comparing the definition of $\Phi_{R}^{\theta}(q ; P)$ with Eq. (5.3), we have

$$
\mathcal{S}(q ; P)=c_{R}^{-1}\left(M_{B}^{2}-P^{2}\right) \Phi_{R}^{\theta}(q ; P) .
$$

The Green's function $S(q ; P)$ is supposed to describe the off-shell coupling of the bound state with its constituent particles, so it must exist for any $P$. From Eqs. (4.4) and (5.4), we see that $S(q ; P)$ satisfies

$$
\begin{aligned}
S_{R}^{-1}\left(q+\frac{1}{2} P\right) S( & (q ; P) S_{R}^{-1}\left(q-\frac{1}{2} P\right) \\
= & \left(M_{B}{ }^{2}-P^{2}\right) c_{R}{ }^{-1} Z_{1 \theta} \theta \\
& +\int \frac{d^{4} q^{\prime}}{(2 \pi)^{4}} I_{R}\left(q, q^{\prime} ; P\right) \mathcal{S}\left(q^{\prime} ; P\right) .
\end{aligned}
$$

Nishijima, Sato, and Ezawa noted that Eq. (5.5) reduces to a homogeneous equation when the BS amplitude diverges at the origin, i.e., when $Z_{1 \theta}$ vanishes. If we assume Fredholm's alternative is valid, the homogeneous equation has solutions only for discrete values of $P^{2}$. Since $S(q ; P)$ must exist for any $P$, they concluded that the BS amplitude cannot diverge at the origin. Thus their basic assumption is the validity of Fredholm's alternative. As we know from the discussion in the preceding section, Fredholm's alternative is not valid when $Z_{1 \theta}$ vanishes; that is, the same homogeneous equation (5.5) describes the continuous spectrum corresponding to $g(q ; P)$ for $P^{2}$ $\neq M_{B}{ }^{2}$ as well as the discrete spectrum corresponding to $S(q ; P)$ at $P^{2}=M_{B}^{2}$ when $Z_{1 \theta}$ vanishes. Furthermore, these discrete spectra are extracted from the continuous spectrum by requiring nor- malizability at least in the asymptotically free theory.

In conclusion, the NSE theorem is valid when the theory is superrenormalizable since $Z_{1 \theta^{-1}}$ is always finite in this case. In the renormalizable theory, however, it should be replaced by the more general results given in Eqs. (3.7) and (3.16).

It is worthwhile noting that the interpolating field constructed by the HNZ method coincides with the elementary boson field $\varphi(x)$ introduced in the Lagrangian when the theory is asymptotically free. This fact, first noted by Nishijima, ${ }^{12}$ can be seen from Eqs. (2.6) and (5.1), which lead to

$\varphi_{c}(x)=\frac{Z_{\theta} \Theta_{R}(x)+a \varphi_{R}(x)+b\left(\square+M_{R}^{2}\right) \varphi_{R}(x)}{Z_{\theta}\left\langle 0\left|\Theta_{R}(0)\right| P\right\rangle+a\left\langle 0\left|\varphi_{R}(0)\right| P\right\rangle}$.

In an asymptotically free theory, $Z_{\theta}$ or $b$ has $\log$ arithmic dependence on $\Lambda$, whereas $a$ has $\Lambda^{2}$ dependence modified by logarithmic factors. Therefore, taking the limit $\Lambda \rightarrow \infty$, we obtain

$$
\varphi_{c}(x)=\varphi_{R}(x),
$$

since $\varphi_{R}(x)$ is normalized by $\left\langle 0\left|\varphi_{R}(0)\right| P\right\rangle=1$. We may see the naturalness of the HNZ construction in this simple result. In more general theories, however, the validity of Eq. (5.7) is not clear because of anomalous dimensions.

\section{ACKNOWLEDGMENTS}

This research was supported in part by the U.S. Department of Energy. It is a pleasure to thank Professor K. Nishijima for useful communications. I wish to thank Professor N. Fuchs for reading the manuscript.

\footnotetext{
*On leave of absence from Department of Physics, University of Tokyo, Tokyo, Japan.

${ }^{1}$ S. J. Brodsky and G. R. Farrar, Phys. Rev. D 11, 1309 (1975); D. Sivers, S. J. Brodsky, and R. Blankenbecler, Phys. Rep. 23C, 1 (1976), and references therein.

${ }^{2}$ See, for example, A. Bastai, L. Bertocchi, S. Fubini, G. Furlan, and M. Tonin, Nuovo Cimento 30, 1912 (1963); T. Appelquist and E. Poggio, Phys. Rev. D 10, 3280 (1974); K. Higashijima, Prog. Theor. Phys. $\underline{52}$,
}

1401 (1974); C. G. Callan, Jr. and D. J. Gross, Phys. Rev. D 11, 2905 (1975); A. H. Guth and D. E. Soper, ibid. 12, 1143 (1975); W. E. Caswell, R. R. Horgan, and G. P. Lepage, SLAC Report No. SLAC- PUB-2066 (unpublished).

${ }^{3}$ K. Nishijima and H. Sato, Prog. Theor. Phys. $\underline{42}, 692$ (1969); Z. F. Ezawa and K. Nishijima, ibid. $\underline{48}, 1751$ (1972).

${ }^{4}$ N. N. Bogoliubov and D. V. Shirkov, Introduction to the 
Theory of Quantized Fields (Interscience, New York, 1959). For a recent review, see R. J. Crewther, in Weak and Electromagnetic Interactions, Cargese, 1975 edited by M. Levy, J.-L. Basdevant, P. Speiser, and R. Gastmans (Plenum, New York, 1976).

${ }^{5}$ C. G. Callan, Jr., Phys. Rev. D 2 1541 (1970); K. Symanzik, Commun. Math. Phys. 18,227 (1970); S. L. Adler, Phys. Rev. D 5 , 3021 (1972).

${ }^{6}$ K. G. Wilson, Phys. Rev. 179, 1499 (1969); W. Zimmermann, in Lectures on Elementary Particles and Quantum Field Theory, edited by S. Deser et al. (MIT Press,
Cambridge, Mass., 1971).

${ }^{7}$ R. Brandt, Ann. Phys. (N.Y.) 52, 122 (1969).

${ }^{8} \mathrm{~K}$. Higashijima and A. Nishimura, Nucl. Phys. B113, 173 (1976) and references therein.

${ }^{9}$ G. Tiktopoulos, J. Math. Phys. 6, 573 (1965):

${ }^{10}$ N. Nakanishi, Prog. Theor. Phys. Suppl. $\underline{43}, 1$ (1969) and references therein.

${ }^{11}$ R. Haag, Phys. Rev. 112 , 669 (1958); K. Nishijima, ibid. 111, 995 (1958); W. Zimmermann, Nuovo Cimento $10,5 \overline{97}$ (1958).

${ }^{12}$ K. Nishijima, Phys. Rev. $\underline{133}$, B204 (1964). 